\title{
Behr syndrome and hypertrophic cardiomyopathy in a family with a novel UCHL1 deletion
}

\author{
Grace McMacken ${ }^{1} \cdot$ Hanns Lochmüller ${ }^{2,3,4} \cdot$ Boglarka Bansagi $^{5} \cdot$ Angela Pyle $^{5} \cdot$ Angela Lochmüller $^{6}$. \\ Patrick F. Chinnery ${ }^{7,8} \cdot$ Steve Laurie $^{3} \cdot$ Sergi Beltran $^{3} \cdot$ Leslie Matalonga $^{3} \cdot$ Rita Horvath $^{7,9}$ (1)
}

Received: 17 May 2020 / Revised: 3 July 2020 / Accepted: 4 July 2020 / Published online: 12 July 2020

(c) The Author(s) 2020

\begin{abstract}
Background Behr syndrome is a clinically distinct, but genetically heterogeneous disorder characterized by optic atrophy, progressive spastic paraparesis, and motor neuropathy often associated with ataxia. The molecular diagnosis is based on gene panel testing or whole-exome/genome sequencing.

Methods Here, we report the clinical presentation of two siblings with a novel genetic form of Behr syndrome. We performed whole-exome sequencing in the two patients and their mother.

Results Both patients had a childhood-onset, slowly progressive disease resembling Behr syndrome, starting with visual impairment, followed by progressive spasticity, weakness, and atrophy of the lower legs and ataxia. They also developed scoliosis, leading to respiratory problems. In their late 30's, both siblings developed a hypertrophic cardiomyopathy and died of sudden cardiac death at age 43 and 40, respectively. Whole-exome sequencing identified the novel homozygous c.627_629del; p.(Gly210del) deletion in UCHL1.

Conclusions The presentation of our patients raises the possibility that hypertrophic cardiomyopathy may be an additional feature of the clinical syndrome associated with UCHL1 mutations, and highlights the importance of cardiac follow-up and treatment in neurodegenerative disease associated with UCHL1 mutations.
\end{abstract}

Keywords Neurogenetics $\cdot$ Behr syndrome $\cdot$ Hereditary spastic paraplegia $\cdot$ Ataxia $\cdot$ Whole exome sequencing

\section{Introduction}

Hereditary spastic paraplegias (HSP) are a clinically and genetically diverse group of disorders characterized by progressive lower limb spasticity. Inheritance can be dominant,

Rita Horvath

rh732@medschl.cam.ac.uk

1 Department of Neurosciences, Royal Victoria Hospital, Belfast, UK

2 Department of Neuropediatrics and Muscle Disorders, Faculty of Medicine, Medical Center - University of Freiburg, Freiburg, Germany

3 Centro Nacional de Análisis Genómico (CNAG-CRG), Center for Genomic Regulation, Barcelona Institute of Science and Technology (BIST), Barcelona, Catalonia, Spain

4 Division of Neurology, Department of Medicine, Children's Hospital of Eastern Ontario Research Institute, The Ottawa Hospital and Brain and Mind Research Institute, University of Ottawa, Ottawa, Canada recessive or X-linked, or rarely mitochondrial [1]. Additional neurological manifestations include optic atrophy, cerebellar ataxia, and peripheral neuropathy [2]. SPG79 is caused by homozygous or compound heterozygous mutations in UCHL1, which encodes Ubiquitin C-terminal hydrolase-L1.

5 Wellcome Centre for Mitochondrial Research, Translational and Clinical Research Institute, Newcastle University, Newcastle upon Tyne, UK

6 GKT School of Medical Education, King's College London, London, UK

7 Department of Clinical Neurosciences, University of Cambridge School of Clinical Medicine, Cambridge Biomedical Campus, Cambridge, UK

8 MRC Mitochondrial Biology Unit, University of Cambridge, Cambridge, UK

9 Department of Clinical Neurosciences, University of Cambridge School of Clinical Medicine, John Van Geest Cambridge Centre for Brain Repair, Robinson Way, Cambridge CB2 OPY, UK 
In addition to spasticity, ataxia, and peripheral neuropathy, SPG79 is characterized by severe optic atrophy in the first decade of life. To date, mutations in UCHL1 have been described in only three families [3-5].

Using whole exome sequencing, we have identified a further family with SPG79, carrying a novel homozygous deletion in UCHL1. Both siblings presented in childhood with motor developmental delay, optic atrophy leading to progressive visual loss, cerebellar ataxia, spastic paraparesis, and motor neuropathy. Intriguingly, both siblings also developed hypertrophic cardiomyopathy, a potentially modifiable clinical feature which has not been described in association with SPG79 before.

\section{Patients and methods}

Patient 1 (P1) and Patient 2 (P2) were born to British parents from the North of England. There is no information on consanguinity. P1 was the product of a normal pregnancy and was born at full-term, and had normal early development. She first presented aged 8 years with visual problems and optic atrophy was detected. Her motor function gradually deteriorated over the next years and she developed progressive lower limb spasticity, foot deformities, and severe scoliosis requiring spinal surgery aged 16 years. Cognitive function was normal. She became non-ambulant following a femoral fracture aged 38 years. From the age of 39, she developed nocturnal hypoventilation and was commenced on overnight non-invasive ventilation.

On examination aged 41 years (Fig. 1a), she had facial dysmorphism including low nasal bridge, micrognathia, and large, low-set ears, bilateral optic atrophy and severely reduced visual acuity, but no ophthalmoparesis or nystagmus. Deep tendon reflexes were absent in the upper and lower limbs, and there was length-dependent atrophy and sensory loss in-keeping with a peripheral neuropathy. She had marked lower limb spasticity, extensor plantar responses and ankle clonus, and a severe scoliosis.

$\mathrm{P} 2$, her younger brother, also presented in early childhood with reduced visual acuity. He developed lower limb spasticity, contractures, and foot deformity. He became nonambulant aged 8 years following surgical correction of his foot deformity. His visual acuity continued to deteriorate due to optic atrophy, so that he was functionally blind. Cognitive function was also unaffected in P2.

Examination of P2 aged 33 (Fig. 1a) revealed bilateral optic atrophy, both pendular and gaze induced nystagmus, ophthalmoparesis, facial dysmorphism including low nasal bridge, micrognathia and large, low-set ears, and facial hypotonia. Deep tendon reflexes were pathologically reduced in upper and lower limbs, and plantar responses were extensor. Sensation was intact. He had severe finger-nose ataxia, dysdiadochokinesia, and truncal ataxia.

Detailed laboratory work-up (including acylcarnitines, urinary organic acids, very long chain fatty acids, pristanic
A

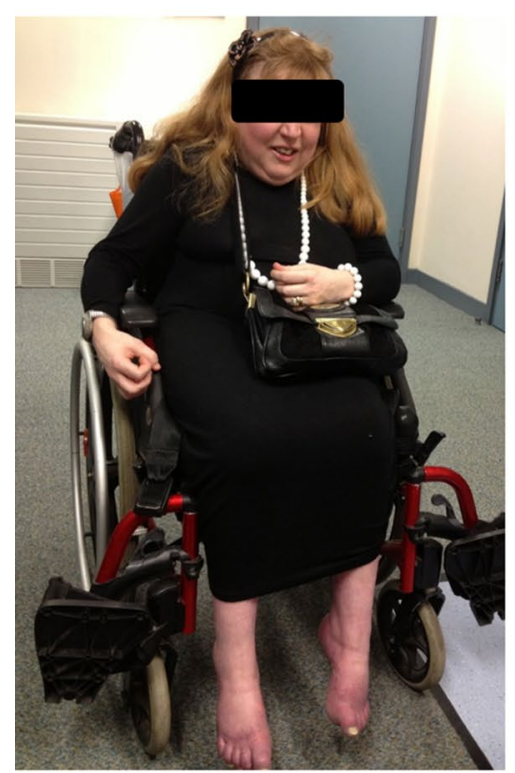

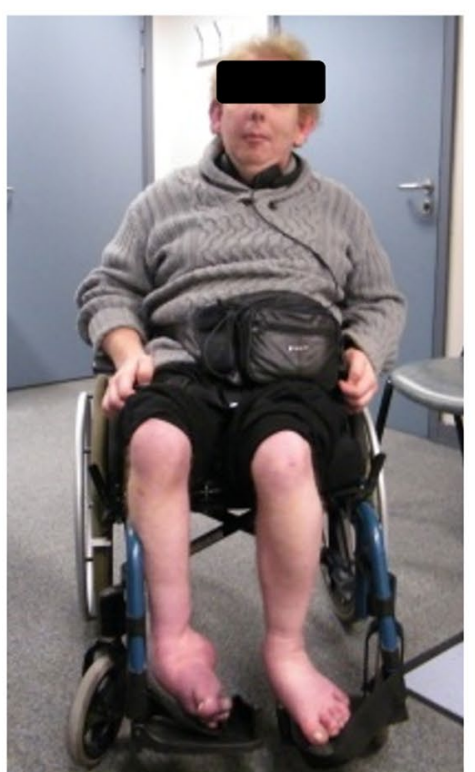

B
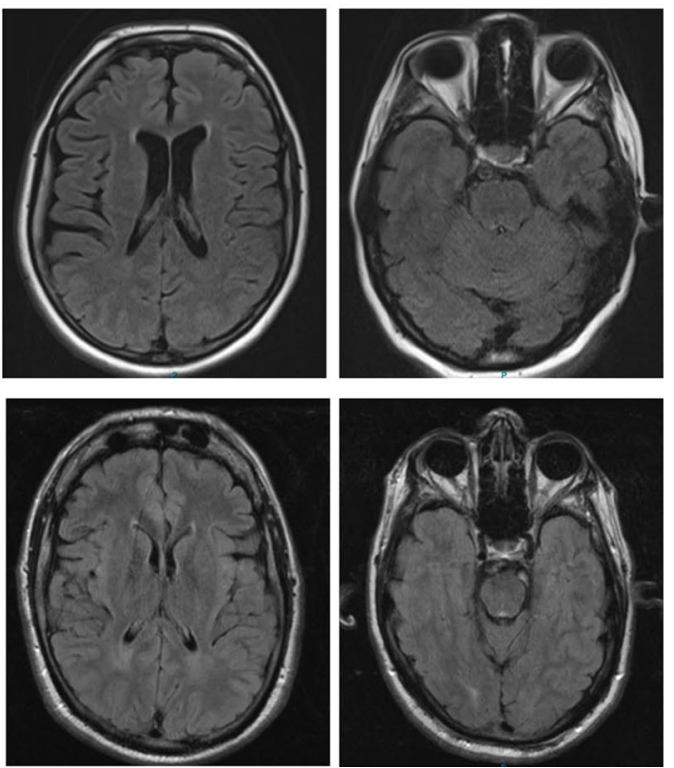

Fig. 1 a P1 aged 40 years (left) and P2 aged 38 years. Both patients have characteristic facial features low-set ears and, and presented with distal upper and lower limb wasting and equinovarus deformity due to peripheral neuropathy and spastic paraparesis. b Axial T2 FLAIR
MR images of P1 (top panels) and P2 (bottom panels). MRI brain in P1 aged 41 years showed mild global atrophy. MRI brain in P2 aged 34 demonstrated no significant abnormality apart from mild increased $\mathrm{T} 2$ signal in the peritrigonal white matter bilaterally 
and phytanic acids, lysosomal enzymes, copper, caeruloplasmin, and acanthocytes) was normal. CSF constituents were normal in both cases. MRI brain in P1 aged 41 years showed mild global atrophy and a small focus of non-specific periventricular T2 signal change at the right frontal horn (Fig. 1b). MRI brain in P2 aged 34 (Fig. 1b) demonstrated no significant abnormality apart from mild increased $\mathrm{T} 2$ signal in the peritrigonal white matter bilaterally. Unfortunately, neurophysiological testing was not technically possible due to the degree of foot deformity and swelling. Direct sequencing of $F X N$ revealed no candidate pathogenic variants. Fifty-six gene inherited peripheral neuropathy panel (Bristol genetics laboratory) which also failed to identify any pathogenic variants and a comparative genomic hybridization (CGH) array was normal.

Given the symptomatic features of a possible mitochondrial disorder, in particular optic atrophy, a cardiac evaluation was carried out in both patients. Echocardiogram in P2 aged 33 years revealed a small, globally hypertrophied leftventricular cavity, left-ventricular systolic ejection gradient (36 $\mathrm{mmHg}$ ), and impaired diastolic left-ventricular function. Echocardiogram in P1 aged 41 years was technically difficult due to severe scoliosis, but also demonstrated cardiomyopathy, with moderately impaired left-ventricular function. The left-ventricular posterior wall was hypokinetic and the interventricular septum was dyskinetic. There was no significant family history of sudden cardiac death, arrhythmias, heart failure, or young stroke.

Both siblings were commenced on ACE inhibitors and beta-blockers and their cardiac function stabilized. Despite this, both siblings developed progressive shortness of breath and apnoeic episodes. P1 died aged 43 years, P2 aged 40 years, both due to sudden cardiac death.

\section{Whole exome sequencing}

WES was performed on genomic DNA in P1 and P2 and on their mother. Their father was deceased at the time of study. The genomic DNA was exome-enriched using Illumina TruSeq $62 \mathrm{Mb}$ exome capture and sequenced on an Illumina HiSeq 2000 with 100 bp paired-end reads. Standard filtering criteria were applied, including minor allele frequency of less than $1 \%$ and high-to-moderate variant effect predictor (i.e., nonsense, splice site, frame-shift, in-frame, and non-synonymous variants). Rare homozygous and compound heterozygous variants were defined, and protein altering and/or putative 'disease causing' mutations, along with their functional annotation, were identified using ANNOVAR. Candidate genes were prioritized if previously associated with a disease phenotype. Genomic and phenotypic data have been submitted to RD-Connect Genome-Phenome Platform (GPAP, https://platform.rd-connect.eu), where they can be accessed under a controlled access agreement.

\section{Detection of homozygous regions}

As part of the RD-Connect GPAP standard analysis pipeline, runs of homozygosity (ROH) were identified in each individual using PLINK v.1.90 [6], applying optimized parameters as defined by Kancheva et al. [7]. Only ROH with a minimum length of $1 \mathrm{Mbp}$ were identified, to exclude common shorter ROH. Consanguinity assessment was performed through application of ROH thresholds for WES as described in Matalonga et al. [8].

\section{Results}

WES analysis revealed the presence of a homozygous c.627_629del; p.(Gly210del) UCHL1 variant (NM_004181.5) in P1 and P2 (Fig. 2a). This variant is reported only once in heterozygous form in gnomAD (allele frequency 0.000004 ), but never in homozygous form. Their unaffected mother was heterozygous for the variant. This variant could disrupt substrate binding at the distal UCHL1 site [9]. Additionally, we assessed IBD regions by the detection of $>1 \mathrm{Mbp}$ runs of homozygosity (ROH). Results have shown that both patients carry an increased number of large $\mathrm{ROH}$ segments, 18 (P1 and P2) versus 5 from their mother, with a total ROH size of $76 \mathrm{MB}$ (P1) and $48 \mathrm{MB}$ (P2) respectively, underlying a possible recent consanguinity (Fig. 2b) [8]. Exome data were also analyzed for variants in genes associated with hypertrophic cardiomyopathy, but no potentially causative variants were detected.

\section{Discussion}

Here, we describe two siblings with clinical presentation comprising progressive spasticity, motor neuropathy, ataxia, and optic atrophy, resembling Behr syndrome, and with the additional clinical finding of hypertrophic cardiomyopathy. Whole exome sequencing identified a novel homozygous deletion of three nucleotides in UCHL1. Paternal data were not available to perform complete segregation studies and confirm his carrier status. However, normal CGH array results excluded a large chromosomal rearrangement in the paternal allele, and although a deletion in UCHL1 cannot be discarded, the possible recent consanguinity identified by $\mathrm{ROH}$ analyses support the homozygous status assumption. Ubiquitin C-terminal hydrolase-L1 (UCHL1) is critically important in maintaining free ubiquitin levels through the addition or removal of ubiquitin to poly-ubiquitin chains, and thus has a central role in cytoplasmic protein degradation [10]. It is one of the most abundant proteins in the nervous system, being highly expressed in both the central and peripheral nervous system [11-13]. Through its close 


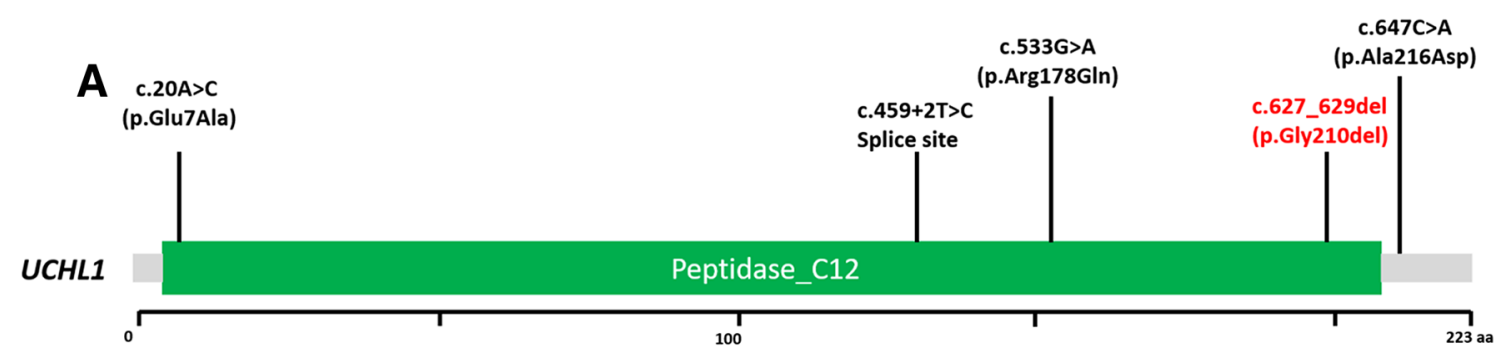

B

\begin{tabular}{|c|c|c|c|c|c|}
\hline & $\begin{array}{c}\text { Number of } \\
\text { runs }\end{array}$ & $\begin{array}{c}\text { Total Sum } \\
\text { ROH (MB) }\end{array}$ & $\begin{array}{c}\text { Median ROH } \\
\text { length (MB) }\end{array}$ & $\begin{array}{c}\text { Longest ROH } \\
\text { fragment (MB) }\end{array}$ & $\begin{array}{c}\text { Size of the run in } \\
\text { chromosome 4 } \\
\text { covering UCHL1 (MB) }\end{array}$ \\
\hline Patient 1 & 18 & 76.2 & 2.6 & 10.2 & 8.8 \\
\hline Patient 2 & 18 & 48.5 & 1.6 & 7.8 & 3.8 \\
\hline Mother & 5 & 11.2 & 1.1 & 3.1 & none \\
\hline
\end{tabular}

\begin{tabular}{|c|c|}
\hline \multicolumn{2}{|c|}{ UCHL1 aa sequence alignment (G at position 210 preserved) } \\
\hline Human (200-220): & CREFTEREQGEVRFSAVALC \\
\hline Rattus norv: & CREFTEREQGEVRFSAVALC \\
\hline Bos taurus: & CREFTEREQGEVRFSAVALC \\
\hline Gallus gallus: & CRQFTEREKGEVRFSAVAFC \\
\hline Danio rerio: & CREFMEREKGEVRFSAVALC \\
\hline
\end{tabular}

Fig. 2 a Pathogenic mutations identified in UCLH1. b Consanguinity analysis through run of homozygosity (ROH) $>1 \mathrm{Mbp}$ detection in autosomal chromosomes. c Conservation of the amino acid affected by the c.627_629del; p.(Gly210del) UCHL1 mutation

interaction with the neuronal cytoskeleton, UCHL1 is thought to have important roles in axonal repair after injury, axonal transport, and synaptic function [14-16].

Mutations in $U C H L 1$ have been described in three families with autosomal recessive spastic paraplegia (SPG79) [3-5]. The phenotype of the patients in the present study had many similarities to the previously reported cases (Table 1). In all cases described to date, the first symptom was of childhood-onset visual loss due to optic atrophy. The subsequent disease course was characterized by varying degrees of spasticity and cerebellar ataxia. Additional, less consistently described features include epilepsy, cognitive impairment, facial myokymia, fasciculations, and reduced peripheral sensation, reflecting the widespread expression of $U C H L 1$ in most neuronal cells. The clinical presentation of our patients and all previously reported cases were compatible with Behr syndrome (Table 1).

First described by Carl Behr in 1909, the classical Behr syndrome (MIM 210000) comprises childhoodonset optic atrophy combined with various neurological symptoms including spastic paraparesis, ataxia, mild ophthalmoparesis, peripheral neuropathy, and a variable degree of learning difficulties [17]. While the optic atrophy in these patients remains stable, other neurological signs progress during childhood becoming more prominent in the second or third decades [17].

Autosomal recessive $O P A 3$ mutations have been identified in Iraqi Jewish patients presenting with Behr syndrome and 3-methylglutaconic aciduria (Costeff syndrome) [18] and more recently autosomal dominant [19] and recessive [20] OPAl mutations were shown in Behr syndrome without metabolic abnormalities. We reported six patients with Behr syndrome and autosomal recessive mutation in C12orf65 [21], and a homozygous C12orf19 mutation has also been reported to cause this phenotype [22], illustrating further genetic heterogeneity of this phenotype. All previously reported genetic forms of Behr syndrome affect mitochondrial proteins involved in different mitochondrial functions. The detection of $U C H L 1$ mutations in this phenotype adds another molecular mechanism, protein de-ubiquitination contributing to Behr 
Journal of Neurology (2020) 267:3643-3649

3647

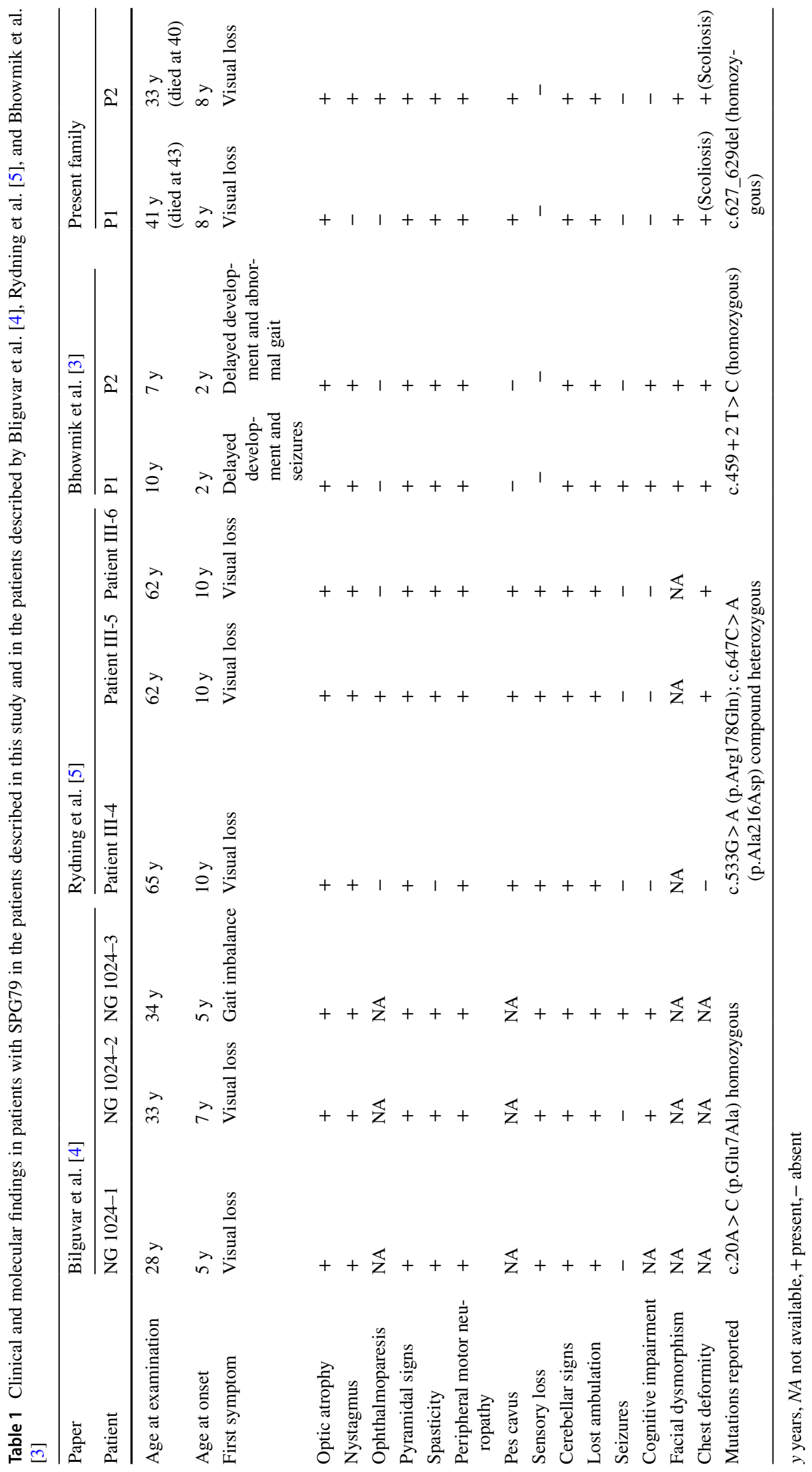

Springer 
syndrome and raising the possibility that this gene is also contributing to mitochondrial protein quality control.

In contrast to previously described cases, however, the siblings in this study both presented with the additional clinical feature of hypertrophic cardiomyopathy. Specifically, both patients demonstrated globally hypertrophied left ventricles in the absence of acquired causes of ventricular dysfunction (e.g., hypertension, myocardial ischaemia, valve dysfunction, prior exposure to toxins, and environmental pathogens). In addition, detailed family history failed to identify other family members known or suspected to be affected by a myocardial disease. The tissue distribution of UCHL1 is predominantly within the nervous system, although it is also expressed at low levels in gonadal cells [23]. Interestingly, UCHL1 is also upregulated in other cells under specialized conditions, for example in fibroblasts during wound healing and in pancreatic, colorectal, medullary thyroid, and breast cancer cells [24-27]. In these conditions, it is suggested that deregulation of the ubiquitin-proteasome system (UPS) may lead to uncontrolled cell growth. Furthermore, the UPS plays a key role in maintaining protein homeostasis and cardiac function, and UCHL1 has been shown to play a key role in this regulation. Knockdown of UCHL1 in cardiomyocytes and mouse hearts rescued cardiac hypertrophy induced by agonist or pressure overload, and overexpression resulted in the opposite effect [28]. We have not carried out functional studies of mutated UCHL1 protein (p.Gly210del). The Glycine residue at position 210 is close to the C-terminus, which regulates protein aggregation and stability [29]. The removal of the final four amino acids from the $\mathrm{C}$-terminus renders the protein insoluble and abolishes binding to ubiquitin substrates [30]. Glycine at position 210 is located just upstream of the final beta-sheet and may have an impact on protein folding and stability. Further studies will be required to ascertain how the c.627_629del; p.(Gly210del) UCHL1 mutation might lead to clinical hypertrophic cardiomyopathy. We recognize that it is not possible to completely exclude an alternative genetic or acquired cause for hypertrophic cardiomyopathy in these siblings, or an unrecognized cardiac disease in previous family members. Nonetheless, the presentation in these cases raises the possibility that hypertrophic cardiomyopathy may be an additional feature of the clinical syndrome associated with UCHL1 mutations. The sudden cardiac death in both patients highlights the importance of cardiac follow-up and treatment in neurodegenerative disease associated with $U C H L 1$ mutations.

In summary, we have reported on a novel UCHLI deletion, resulting in a progressive neurodegenerative syndrome. The additional feature of hypertrophic cardiomyopathy has not been previously described. Cardiac evaluation is, therefore, essential in cases with this rare neurological phenotype.
Author contributions GM has collected clinical data and drafted the manuscript. HL and AP performed bioinformatics analysis of exome sequencing data. BB and PFC performed clinical follow-up examination of the patients. AL collected clinical information and drafted Table 1; SL, SB, and LM performed the homozygosity analysis and participated in drafting the manuscript; $\mathrm{RH}$ performed clinical followup examinations of the patients, initiated genetic testing, and drafted the manuscript.

Funding The study was supported by the Newton Fund UK/Turkey (MR/N027302/1 to HL and RH), the Medical Research Council (UK) (MR/N025431/1 to RH), the Wellcome Investigator fund (109915/Z/15/Z to RH), the Lily Foundation UK (to RH), the European Research Council (309548 to RH), and the Wellcome Trust Pathfinder Scheme (201064/Z/16/Z to HL and RH). HL receives support from the Canadian Institutes of Health Research (Foundation Grant FDN-167281), the Canadian Institutes of Health Research and Muscular Dystrophy Canada (Network Catalyst Grant for NMD4C), the Canada Foundation for Innovation (CFI-JELF 38412), and the Canada Research Chairs program (Canada Research Chair in Neuromuscular Genomics and Health, 950-232279). The study was further supported by the Horizon 2020 research and innovation program via grant 779257 "Solve-RD". Data were analyzed using the RD-Connect GenomePhenome Analysis platform developed under FP7/2007-2013 funded project (grant agreement no. 305444).

Data availability Whole exome sequencing data of the patients and their mother have been uploaded to the RD-CONNECT platform for data sharing and storage.

\section{Compliance with ethical standards}

Conflict of interest The authors have no conflict of interest.

Ethics approval Informed consent was obtained from each participant approved by the Yorkshire and The Humber-Leeds Bradford Research Ethics Committee (13/YH/0310).

Consent to participate We obtained informed consent from both patients and their mother for this study.

Consent for publication The patients died, therefore we could not obtain consent for this publication.

Open Access This article is licensed under a Creative Commons Attribution 4.0 International License, which permits use, sharing, adaptation, distribution and reproduction in any medium or format, as long as you give appropriate credit to the original author(s) and the source, provide a link to the Creative Commons licence, and indicate if changes were made. The images or other third party material in this article are included in the article's Creative Commons licence, unless indicated otherwise in a credit line to the material. If material is not included in the article's Creative Commons licence and your intended use is not permitted by statutory regulation or exceeds the permitted use, you will need to obtain permission directly from the copyright holder. To view a copy of this licence, visit http://creativecommons.org/licenses/by/4.0/. 


\section{References}

1. Finsterer J, Löscher W, Quasthoff S, Wanschitz J, Auer-Grumbach M, Stevanin G (2012) Hereditary spastic paraplegias with autosomal dominant, recessive, $\mathrm{X}$-linked, or maternal trait of inheritance. J Neurol Sci 318:1-18

2. Parodi L, Fenu S, Stevanin G, Durr A (2017) Hereditary spastic paraplegia: more than an upper motor neuron disease. Rev Neurol (Paris) 173:352-360

3. Bhowmik AD, Patil SJ, Deshpande DV, Bhat V, Dalal A (2018) Novel splice-site variant of UCHL1 in an Indian family with autosomal recessive spastic paraplegia-79. J Hum Genet 63:927

4. Bilguvar K, Tyagi NK, Ozkara C, Tuysuz B, Bakircioglu M, Choi M, Delil S, Caglayan AO, Baranoski JF, Erturk O et al (2013) Recessive loss of function of the neuronal ubiquitin hydrolase UCHL1 leads to early-onset progressive neurodegeneration. Proc Natl Acad Sci U S A 110:3489-3494

5. Rydning SL, Backe PH, Sousa MML, Iqbal Z, Øye A-M, Sheng Y, Yang M, Lin X, Slupphaug G, Nordenmark TH et al (2017) Novel UCHL1 mutations reveal new insights into ubiquitin processing. Hum Mol Genet 26:1031-1040

6. Purcell S, Neale B, Todd-Brown K, Thomas L, Ferreira MA, Bender D, Maller J, Sklar P, de Bakker PI, Daly MJ, Sham PC (2007) PLINK: a tool set for whole genome association and population-based linkage analyses. Am J Hum Genet 81:559-575

7. Kancheva D, Atkinson D, De Rijk P, Zimon M, Chamova T, Mitev V, Yaramis A, Maria Fabrizi G, Topaloglu H, Tournev I, Parman Y, Parma Y, Battaloglu E, Estrada-Cuzcano A, Jordanova A (2016) Novel mutations in genes causing hereditary spastic paraplegia and Charcot-Marie-Tooth neuropathy identified by an optimized protocol for homozygosity mapping based on wholeexome sequencing. Genet Med 18(6):600-607

8. Matalonga L, Laurie S, Papakonstantinou A, Piscia D, Mereu E, Bullich G, Thompson R, Horvath R, Pérez-Jurado L, Riess O et al (2020) Improved diagnosis of rare disease patients through systematic detection of runs of homozygosity. J Mol Diagn. https ://doi.org/10.1016/j.jmoldx.2020.06.008

9. Boudreaux DA, Maiti TK, Davies CW, Das C (2010) Ubiquitin vinyl methyl ester binding orients the misaligned active site of the ubiquitin hydrolase UCHL1 into productive conformation. Proc Natl Acad Sci U S A 107:9117-9122

10. Liu Y, Fallon L, Lashuel HA, Liu Z, Lansbury PT (2002) The UCH-L1 gene encodes two opposing enzymatic activities that affect $\alpha$-synuclein degradation and Parkinson's disease susceptibility. Cell 111:209-218

11. Ann ES, Mizoguchi A, Okajima S, Ide C (1994) Motor axon terminal regeneration as studied by protein gene product $9.5 \mathrm{immu}-$ nohistochemistry in the rat. Arch Histol Cytol 57:317-330

12. Doran JF, Jackson P, Kynoch PA, Thompson RJ (1983) Isolation of PGP 9.5, a new human neurone-specific protein detected by high-resolution two-dimensional electrophoresis. J Neurochem 40:1542-1547

13. Lin WM, Hsieh ST, Huang IT, Griffin JW, Chen WP (1997) Ultrastructural localization and regulation of protein gene product 9.5. NeuroReport 8:2999-3004

14. Chen F, Sugiura Y, Myers KG, Liu Y, Lin W (2010) Ubiquitin carboxyl-terminal hydrolase L1 is required for maintaining the structure and function of the neuromuscular junction. Proc Natl Acad Sci U S A 107:1636-1641

15. Genç B, Jara JH, Schultz MC, Manuel M, Stanford MJ, Gautam M, Klessner JL, Sekerkova G, Heller DB, Cox GA et al (2016) Absence of UCHL 1 function leads to selective motor neuropathy. Ann Clin Transl Neurol 3:331-345
16. Gong B, Cao Z, Zheng P, Vitolo OV, Liu S, Staniszewski A, Moolman D, Zhang H, Shelanski M, Arancio O (2006) Ubiquitin hydrolase Uch-L1 rescues beta-amyloid-induced decreases in synaptic function and contextual memory. Cell 126:775-788

17. Behr C (1909) Die komplizierte, hereditär-familiäre optikusatrophie des kindesalters: ein bisher nicht beschriebener symptomkompleks. Klinische Monatsblätter für Augenheilkunde 47:138-160

18. Anikster Y, Kleta R, Shaag A, Gahl WA, Elpeleg O (2001) Type III 3-methylglutaconic aciduria (optic atrophy plus syndrome, or Costeff optic atrophy syndrome): identification of the OPA3 gene and its founder mutation in Iraqi Jews. Am J Hum Genet 69:1218-1224

19. Marelli C, Amati-Bonneau P, Reynier P, Layet V, Layet A, Stevanin G, Brissaud E, Bonneau D, Durr A, Brice A (2011) Heterozygous OPA1 mutations in Behr syndrome. Brain 134:e169

20. Bonneau D, Colin E, Oca F, Ferré M, Chevrollier A, Guéguen N, Desquiret-Dumas V, N'Guyen S, Barth M, Zanlonghi X et al (2014) Early-onset Behr syndrome due to compound heterozygous mutations in OPA1. Brain 137:e301

21. Pyle A, Ramesh V, Bartsakoulia M, Boczonadi V, Gomez-Duran A, Herczegfalvi A, Blakely EL, Smertenko T, Duff J, Eglon G, Moore D, Yu-Wai-Man P, Douroudis K, Santibanez-Koref M, Griffin H, Lochmüller H, Karcagi V, Taylor RW, Chinnery PF, Horvath R (2014) Behr's Syndrome is typically associated with disturbed mitochondrial translation and mutations in the C12orf65 gene. J Neuromuscul Dis 1(1):55-63

22. Kleffner I, Wessling C, Gess B, Korsukewitz C, Allkemper T, Schirmacher A, Young P, Senderek J, Husstedt IW (2015) Behr syndrome with homozygous C19ORF12 mutation. J Neurol Sci 357(1-2):115-118

23. Wilkinson KD, Deshpande S, Larsen CN (1992) Comparisons of neuronal (PGP 9.5) and non-neuronal ubiquitin C-terminal hydrolases. Biochem Soc Trans 20:631-637

24. Miyoshi Y, Nakayama S, Torikoshi Y, Tanaka S, Ishihara H, Taguchi T, Tamaki Y, Noguchi S (2006) High expression of ubiquitin carboxy-terminal hydrolase-L1 and -L3 mRNA predicts early recurrence in patients with invasive breast cancer. Cancer Sci 97:523-529

25. Takano T, Miyauchi A, Matsuzuka F, Yoshida H, Nakata Y, Kuma K, Amino N (2004) PGP9.5 mRNA could contribute to the molecular-based diagnosis of medullary thyroid carcinoma. Eur J Cancer Oxf Engl 40:614-618

26. Tezel E, Hibi K, Nagasaka T, Nakao A (2000) PGP9.5 as a prognostic factor in pancreatic cancer. Clin Cancer Res Off $\mathbf{J}$ Am Assoc Cancer Res 6:4764-4767

27. Yamazaki T, Hibi K, Takase T, Tezel E, Nakayama H, Kasai Y, Ito K, Akiyama S, Nagasaka T, Nakao A (2002) PGP9.5 as a marker for invasive colorectal cancer. Clin Cancer Res Off J Am Assoc Cancer Res 8:192-195

28. Bi HL, Zhang XL, Zhang YL, Xie X, Xia YL, Du J, Li HH (2020) The deubiquitinase UCHL1 regulates cardiac hypertrophy by stabilizing epidermal growth factor receptor. Sci Adv 6(16):eaax4826

29. Bishop P, Rocca D, Henley JM (2016) Ubiquitin C-terminal hydrolase L1 (UCH-L1): structure, distribution and roles in brain function and dysfunction. Biochem J 473(16):2453-2462

30. Bishop P, Rubin P, Thomson AR, Rocca D, Henley JM (2014) The ubiquitin C-terminal hydrolase L1 (UCH-L1) C-terminus plays a key role in protein stability, but its farnesylation is not required for membrane association in primary neurons. J Biol Chem 289(52):36140-36149 